\title{
A SHORT NOTE ON TWO INEQUALITIES FOR SINE POLYNOMIALS
}

\section{HORST ALZER}

Abstract. We present elementary proofs for

$$
\sum_{\nu=1}^{n}(n+1-\nu) \sin (\nu x)>0
$$

due to Lukács, and for

$$
\sum_{\nu=1}^{n} \sin (\nu x)+\frac{1}{2} \sin ((n+1) x) \geq 0
$$

due to Feje̊r. Both inequalities are valid for $x \in(0, \pi)$ and $n=1,2, \ldots$ Furthermore we determine all cases of equality in (*).

The subject of this paper are the trigonometric inequalities

$$
\sum_{\nu=1}^{n}(n+1-\nu) \sin (\nu x)>0
$$

and

$$
\sum_{\nu=1}^{n} \sin (\nu x)+\frac{1}{2} \sin ((n+1) x) \geq 0
$$

which are both valid for $x \in(0, \pi)$ and $n=1,2, \ldots$ Inequality (1) was discovered by F. Lukács who informed L. Fejér in a private communication about his result,

Received September 2, 1991. 
and in 1928 Fejér [1, pp. 335-339] provided elegant (but not simple) proofs for (1) as well as (2) by showing that the sums on the left-hand side of (1) and (2) are coefficients of special power series with non-negative coefficients.

The aim of this note is to present elementary short proofs for inequalities (1) and (2). Furthermore we determine all cases of equality in (2); this result seems to be new.

Let $x \in(0, \pi)$ and $n \geq 1$. We define

$$
t_{n}(x)=\sum_{\nu=1}^{n} \sin (\nu x)
$$

then we obtain

$$
\begin{aligned}
t_{n}(x)+t_{n+1}(x) & =2 \sum_{\nu=1}^{n} \sin (\nu x)+\sin ((n+1) x) \\
& =\frac{1}{\sin \frac{x}{2}}\left[\cos \frac{x}{2}-\cos \left(\left(n+\frac{1}{2}\right) x\right)\right]+\sin ((n+1) x),
\end{aligned}
$$

and because of

$$
\cos \left(\left(n+\frac{1}{2}\right) x\right)=\cos ((n+1) x) \cos \frac{x}{2}+\sin ((n+1) x) \sin \frac{x}{2}
$$

we get

$$
t_{n}(x)+t_{n+1}(x)=\cot \frac{x}{2}[1-\cos ((n+1) x)] \geq 0,
$$

which proves inequality (2). Furthermore we conclude that the sign of equality holds in (2) if and only if $x=\frac{2 \pi k}{n+1}, 0<k<\frac{n+1}{2}(k \in \mathbb{Z})$.

If we define

$$
s_{n}(x)=\sum_{\nu=1}^{n}(n+1-\nu) \sin (\nu x),
$$

then we have

$$
s_{n}(x)=\sum_{\nu=1}^{n} t_{\nu}(x) \text {. }
$$

We consider two cases: If $n$ is odd, say $n=2 m+1(m \geq 0)$, then we obtain from (3) and (4):

$$
s_{2 m+1}(x)=t_{1}(x)+\sum_{\nu=1}^{m}\left[t_{2 \nu}(x)+t_{2 \nu+1}(x)\right]>0 .
$$


And if $n$ is even, say $n=2 m(m \geq 1)$, then we get

$$
\begin{aligned}
s_{2 m}(x) & =\sum_{\nu=1}^{m}\left[t_{2 \nu-1}(x)+t_{2 \nu}(x)\right] \\
& \geq t_{1}(x)+t_{2}(x)=\cot \frac{x}{2}[1-\cos (2 x)]>0 .
\end{aligned}
$$

This establishes inequality (1).

Finally we mention two interesting results which are closely connected to Lukács' inequality. In 1954 A. H. Tureckii [4] (see also [3, p. 252]) published a kind of converse of inequality (1):

$$
\sum_{\nu=1}^{n}(n+1-\nu)|\sin (\nu x)| \leq \frac{(n+1)^{2}}{\pi}, \quad x \in \mathbb{R}, n=1,2, \ldots,
$$

and in 1970 the following striking companion of (5) was proved by J. B. Kelly [2]:

$$
\sum_{\nu=1}^{n}(-1)^{\nu+1}(n+1-\nu)|\sin (\nu x)| \geq 0, \quad x \in \mathbb{R}, n=1,2, \ldots
$$

\section{References}

[1] L. Fejér, "Einige Sãtze, die sich auf das Vorzeichen einer ganzen rationalen Funktion beziehen; nebst Anwendungen dieser Sätze auf die Abschnitte und Abschnittsmittelwerte von ebenen und räumlichen harmonischen Entwicklungen und von beschränkten Potenzreihen", Monatsh. Math. Phys. 35 (1928), 305-344.

[2] J. B. Kelly, "Metric inequalities and symmetric differences", in: Inequalities II (0. Shisha, ed.), 193-212, Academic Press, New York, 1970.

[3] D. S. Mitrinović, "Analytic Inequalities", Springer-Verlag, New York, 1970.

[4] A. H. Tureckii., "On a function deviating least from zero", (Russian), Belorussk. Gos. Univ. Uc. Zap. Ser. Fiz.-Mat. 16 (1954), 41-43. 